\title{
Análise da participação feminina nos cursos técnicos e de graduação da área de Informática da Rede Federal de Educação Tecnológica e do Cefet/RJ campus Nova Friburgo
}

\author{
Gisele Moraes Marinho ${ }^{1}$, Simone Tardin Fagundes ${ }^{1}$, Carolina de Lima Aguilar ${ }^{1}$ \\ ${ }^{1}$ Centro Federal de Educação Tecnológica Celso Suckow da Fonseca \\ gisele.marinho@cefet-rj.br, simone.fagundes@cefet-rj.br \\ carolina.aguilarecefet-rj.br
}

\begin{abstract}
Several studies pointed women as minory in the higher courses of Technology, however there are few studies that investigate their participation in technical courses. This paper presents an overview of the participation of women in higher education, as well as in the professional technical courses of the Brazilian Professional, Scientific and Technological Education Network and in particular of the courses offered by Cefet/RJ campus Nova Friburgo. Aspects related to admission and the academic situation of the students of these courses are discussed.
\end{abstract}

\begin{abstract}
Resumo. Diversos estudos apontam que as mulheres são minoria nos cursos superiores da área de Tecnologia da Informação, no entanto há poucos trabalhos que investigam sua participação em cursos técnicos. Este trabalho apresenta um panorama da participação feminina nos cursos superiores de Bacharelado e Tecnologia, bem como nos cursos técnicos da Rede Federal de Educação Profissional, Científica e Tecnológica e em especial dos cursos ofertados pelo Cefet/RJ campus Nova Friburgo. São discutidos aspectos relacionados ao ingresso e à situação academia das estudantes desses cursos.
\end{abstract}

\section{Introdução}

A presença feminina nos cursos de Ciência e Tecnologia vem sendo investigada e discutida ao longo dos últimos anos. A desproporcionalidade entre mulheres e homens nesse ramo é considerada evidente entre os pesquisadores da área [Hill et al. 2010].

Apesar da maior presença das mulheres no ensino superior e na pós-graduação, suas escolhas disciplinares não se modificaram significativamente. De acordo com [Tabak 2002], as mulheres são encontradas predominantemente em áreas consideradas femininas, como educação e saúde, e os homens continuam a ser maioria nas ciências exatas, como as engenharias. Na área de Informática ou Ciência da Computação há diversos estudos que indicam que as mulheres são minoria nos cursos superiores e nas pós-graduações em todo o mundo e igualmente no Brasil [Monard and Fortes 2013, NCWIT 2016].

Dentre os fatores apontados para tal ocorrência, destaca-se o fato de o processo de educação das mulheres estar geralmente atrelado a papéis sociais determinados em bases biológicas e uma forte influência cultural exercida pela mídia, escolas e pela própria família. 
Um outro fator importante é a falta de representação feminina na área: muitas mulheres participaram da construção da história da computação, porém os nomes dos homens são sempre os mais citados e, dificilmente, uma pessoa que não é especialista na área tem conhecimento dos nomes e dos papéis que exerceram essas mulheres.

Tal comportamento se reflete também nas escolas voltadas à formação de nível técnico. De acordo com [Alves 2016], o acesso à cursos técnicos que exigem um domínio considerável das tecnologias e engenharias ainda é um fator de exclusão. Assim como ocorre nos cursos de Graduação e Pós-Graduação, a atividade feminina em profissões de nível técnico se distancia de algumas áreas e se concentra em determinados setores como serviços pessoais, saúde e educação.

Cabe ressaltar que nas últimas décadas o Brasil vivenciou a maior expansão de sua história da rede de Educação Profissional. Criada em 2008 pela Lei $n^{0} 11.892$ a Rede Federal de Educação Profissional, Científica e Tecnológica (RFEPCT) constituiuse em um marco na ampliação, interiorização e diversificação da educação profissional e tecnológica no país. Em 2018, considerando os campi associados a essas instituições, tem-se ao todo 659 unidades distribuídas entre as 27 unidades federadas do país.

Sob este contexto, este trabalho tem como objetivo apresentar um panorama da participação feminina nos cursos superiores de Bacharelado e Tecnologia, bem como nos cursos técnicos da Rede Federal de Educação Profissional, Científica e Tecnológica e em especial dos cursos ofertados pelo Cefet/RJ campus Nova Friburgo.

Para tanto, este artigo está organizado da seguinte forma: a seção 2 descreve as origens dos dados coletados, a seção 3 apresenta a análise dos dados categorizados em Ensino Superior e Ensino Técnico e, por fim, a seção 4 trata das considerações finais e trabalhos futuros.

\section{Metodologia}

A coleta dos dados utilizados nesta análise se deu em duas etapas. Na primeira etapa foram coletados os dados disponibilizados na Plataforma Nilo Peçanha referentes ao número de matrícula, no ano de 2017, por sexo nos cursos de Graduação de Bacharelado e de Tecnologia e nos cursos técnicos ofertados nas modalidades subsequente, concomitante e integrada ao Ensino Médio classificados no Eixo Tecnológico Informação e Comunicação e subeixo Tecnológico Informática.

A Plataforma Nilo Peçanha (PNP), criada em 2017, é um ambiente virtual do Governo Federal de coleta, validação e disseminação das estatísticas oficiais da RFEPCT e objetiva reunir dados relativos ao corpo docente, discente, técnico-administrativo e de gastos financeiros [Brasil 2018].

Na segunda etapa, foram coletados os dados referentes ao número de ingressantes e à situação das matrículas dos alunos dos cursos do Cefet/RJ campus Nova Friburgo, conforme classificação da etapa anterior e tendo como base o segundo semestre de 2018. Os dados foram obtidos através do SIE (Sistema de Informações para o Ensino), sistema disponibilizado para uso administrativo na instituição. Dessa forma, analisaram-se os seguintes indicadores:

- Número de ingressantes por semestre desde o primeiro semestre/ano de oferta até o ano de 2018, para os cursos ainda ativos, ou até o último período de oferta de 
novas vagas, para os cursos extintos.

- Número de estudantes sem evasão, ou seja, alunos com matrícula ativa.

- Número de estudantes com matrícula trancada.

- Número de estudantes com matrícula encerrada, incluindo cancelamentos, abandonos e transferências.

- Número de estudantes formados.

\section{Análise dos dados}

Após a coleta dos dados, as tabelas foram agrupadas conforme nível do curso e tipo de evento para a descrição das análises nas seções a seguir.

\subsection{Cursos de Graduação}

Os dados disponibilizados na Plataforma Nilo Peçanha apontam que 374 unidades de ensino ofertam cursos superiores de Tecnologia e/ou Bacharelado na Rede Profissional de Educação Tecnológica, totalizando 1.179 cursos e 178.323 matrículas. Desses, aproximadamente $22 \%$ dos cursos (263) e $20 \%$ das matrículas (35.282) estão inseridos no eixo/subeixo tecnológico analisado.

O primeiro curso superior da área ofertado pelo Cefet/RJ no campus Maracanã foi o curso superior de Tecnologia em Sistemas para Internet no primeiro semestre de 1998. Atualmente, este curso encontra-se em processo de extinção. Em 2012 foi criado, no mesmo campus, o curso superior de Bacharelado em Ciência da Computação com oferta semestral de 40 vagas.

Com a expansão da rede, foram abertos dois novos cursos no ano de 2014: Bacharelado em Sistemas de Informação, no campus Nova Friburgo, e Bacharelado em Engenharia de Computação, no campus Petrópolis, com oferta semestral de 40 e 25 vagas, respectivamente.

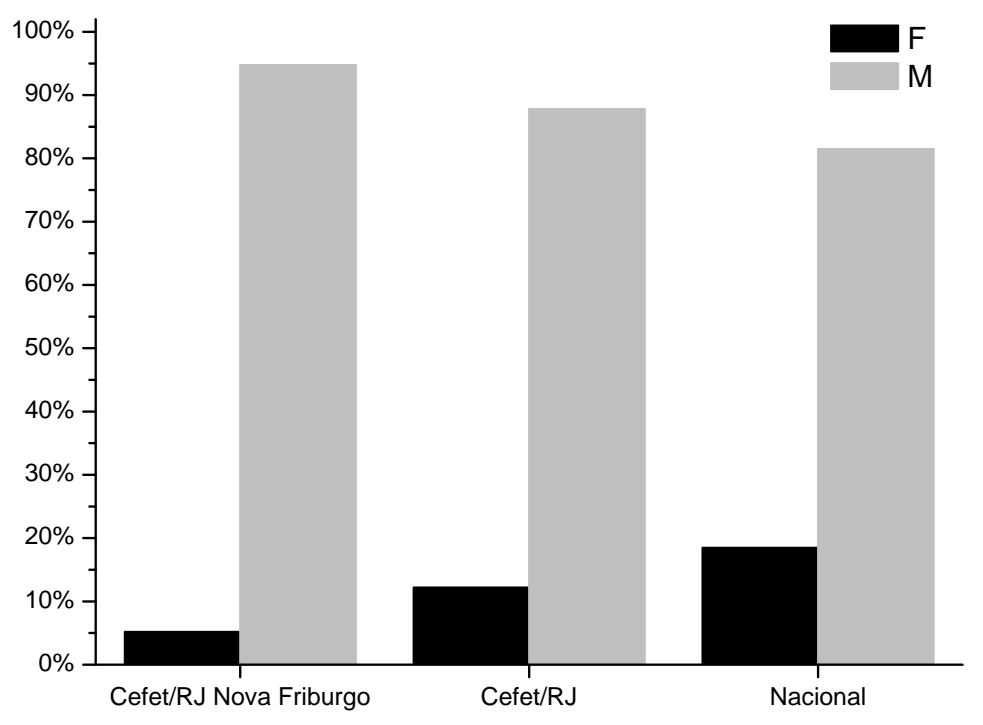

Figura 1. Percentual de alunos matriculados nos cursos de graduação no ano de 2017, Eixo Tecnológico Informação e Comunicação/Informática 
O gráfico da Figura 1 apresenta uma comparação entre o percentual do número de matrículas, por sexo, nos cursos de Tecnologia e Bacharelado do eixo analisado a nível nacional, institucional e apenas no campus Nova Friburgo, através dos dados obtidos na Plataforma Nilo Peçanha.

Pode-se observar que a participação das mulheres nos cursos analisados é ainda tímida. O Cefet/RJ segue a tendência nacional já observada em outras pesquisas como a de [Mendes and da Silva Figueiredo 2015], que analisou a presença feminina no curso de Sistemas de Informação da Universidade Federal do Mato Grosso (UFMT), a de [Moreira et al. 2016], que mostrou o percentual de alunas matriculadas nos cursos da área de Computação da Universidade Federal da Paraíba (UFPB), a de [Santos 2017] que investigou a participação feminina nos cursos de Licenciatura em Computação e a de [de Sousa et al. 2016], que analisou os cursos de Ciência da Computação e Engenharia da Computação no Brasil e na Universidade do Vale do Itajaí (Univali) em Santa Catarina. Esses levantamentos, realizados em diferentes regiões do país, apontam o caráter nacional dessa tendência, uma vez que não há grandes variações nas estatísticas apresentadas.

Com relação ao campus Nova Friburgo, observa-se uma proporção ainda menor de mulheres matriculadas no curso de Sistemas de Informação, correspondendo apenas a $5 \%$ do total de matrículas.

O curso de Sistemas de Informação do campus Nova Friburgo iniciou suas atividades no $1^{\circ}$ semestre de 2014. Do total de vagas ofertadas, apenas 3 foram preenchidas por estudantes do sexo feminino naquele semestre. Ao longo dos anos, o percentual de mulheres ingressantes não teve grandes variações. $\mathrm{O}$ auge de ingresso de mulheres no curso ocorreu no primeiro semestre do ano de 2018, totalizando $20 \%$ do total, conforme pode-se observar na Figura 2.

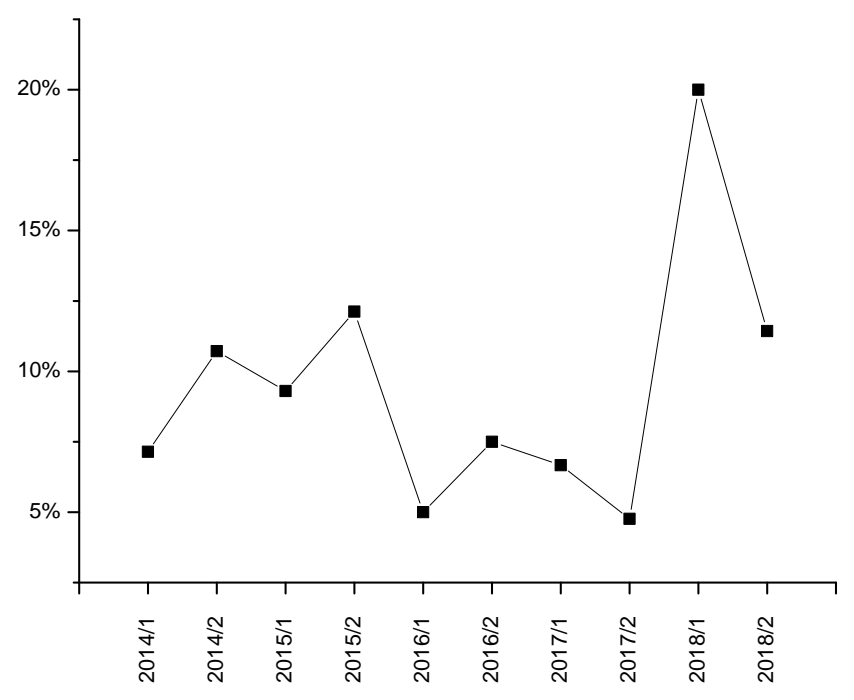

Figura 2. Percentual de mulheres ingressantes no curso de Sistemas de Informação do Cefet/RJ campus Nova Friburgo

Atualmente, as mulheres representam pouco mais de $10 \%$ do total de alunos matriculados e, historicamente, as matrículas femininas somam um total de $9 \%$ de todas as 
matrículas do curso. Estes dados demonstram uma semelhança muito expressiva com a análise apresentada por [Mendes and da Silva Figueiredo 2015] sobre o curso de Sistemas de Informação da UFMT, cujos percentuais eram os mesmos até o primeiro semestre de 2015.

Apresenta-se agora uma análise da situação da matrícula dos alunos do curso de Sistemas de Informação no segundo semestre de 2018 através dos dados coletados no SIE.

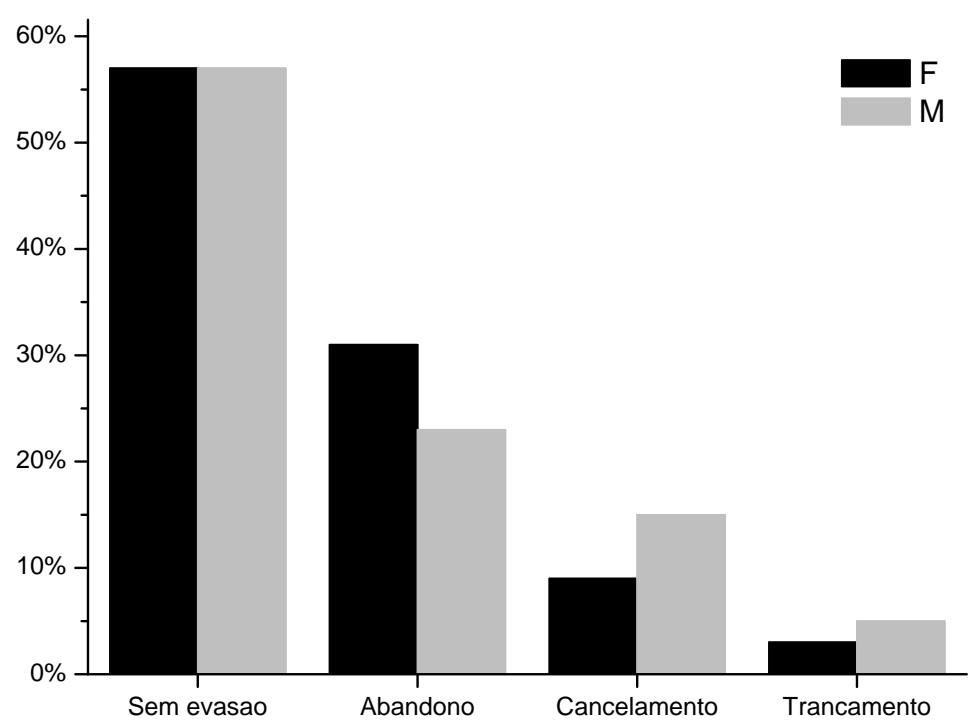

Figura 3. Percentual relativo de alunos por situação de matrícula no ano de 2018

Os percentuais exibidos no gráfico da Figura 3 referem-se à uma análise relativa entre o número de estudantes em cada situação dividido pelo total de estudantes agrupados por sexo. Por exemplo, do total das 35 alunas que já ingressaram no curso, 20 permanecem com matrícula ativa (coluna Sem evasão), correspondendo a um percentual de $57 \%$. É possível observar que o percentual de alunos de ambos os sexos que permanecem no curso é o mesmo. O percentual de cancelamento e de trancamento entre o público feminino é menor que o observado entre o público masculino, porém o percentual de abandono é maior entre as mulheres. No gráfico foi omitido o número de formandos uma vez que, por se tratar de um curso recente, o número de formandos, ainda pequeno, não geraria uma estatística apropriada para o objetivo desta análise.

\subsection{Ensino Técnico}

De acordo com os dados coletados na Plataforma Nilo Peçanha, 590 unidades de ensino ofertam cursos ténicos nas modalidades concomitante e subsequente, totalizando 3.914 cursos e 323.925 matrículas. Desses, aproximadamente 15\% dos cursos (580) e 13\% das matrículas (41.466) estão inseridos no eixo/subeixo tecnológico analisado. Em relação aos cursos técnicos ofertados na modalidade integrada ao Ensino Médio, existem 510 unidades ofertando 1.956 cursos e totalizando 223.893 matrículas, entre os quais aproximadamente $24 \%$ dos cursos (470) e $21 \%$ das matrículas (47.439) pertencem ao eixo em questão.

A ideia do curso técnico de Informática no Cefet/RJ originalmente nasceu como uma especialização do Curso Técnico de Eletrônica no ano de 1998. A oficialização do 
curso Técnico de Informática como um curso independente aconteceu no ano de 2000 no campus Maracanã, oferecido na modalidade subsequente. Ao longo do tempo os cursos foram sendo reformulados, modificando as modalidades de oferta (concomitante, subsequente e integrado), ao mesmo tempo em que outros cursos técnicos iam surgindo, incluindo cursos ofertados na modalidade à Distância. Atualmente, no eixo analisado, são ofertados cursos integrados nos campi Maracanã, Nova Friburgo e Nova Iguaçu.

O campus Nova Friburgo iniciou suas atividades no segundo semestre de 2008 com dois cursos de Graduação e o curso técnico de Informática Industrial ofertado até o primeiro semestre de 2010. No segundo semestre desse mesmo ano, o curso passou por uma reformulação e, no primeiro semestre de 2011, foi ofertada a primeira turma do curso técnico de Informática. Ambos os cursos foram oferecidos na modalidade de concomitância externa. No ano de 2015, foi implementado o curso Técnico de Informática Integrado ao Ensino Médio, dando continuidade ao trabalho iniciado com o curso concomitante ofertado até o primeiro semestre de 2014.

O gráfico da Figura 4 apresenta uma análise do perfil dos ingressantes nos cursos técnicos de Informática Industrial e Informática ofertados na modalidade concomitante no campus Nova Friburgo.

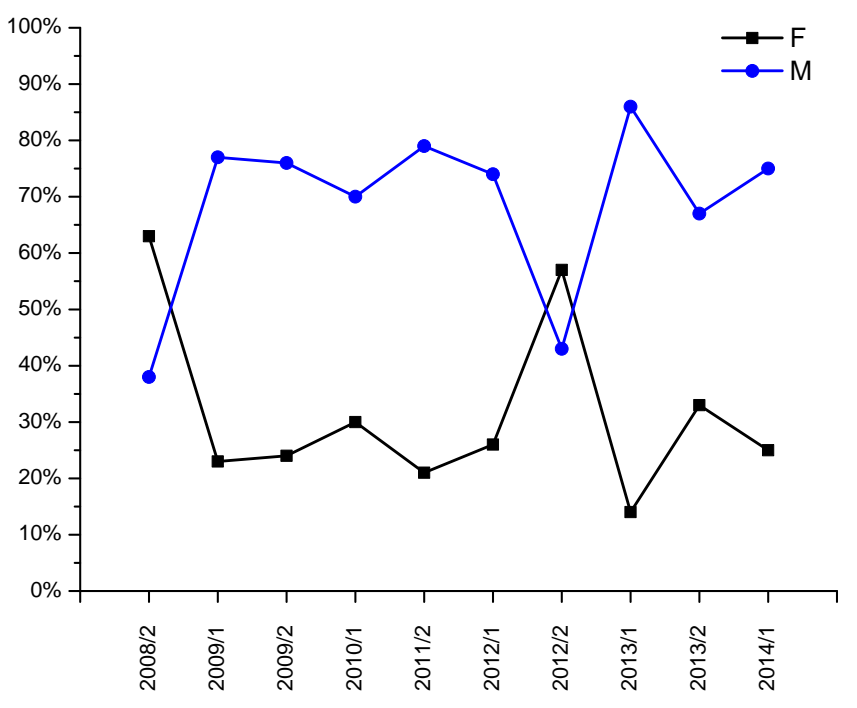

Figura 4. Percentual de ingressantes por sexo no curso Técnico de Informática concomitante do campus Nova Friburgo

No primeiro semestre do curso, observa-se que o número de ingressantes mulheres foi maior do que o de homens. Com o passar do tempo, a entrada manteve-se majoritariamente masculina, com uma média de apenas $31 \%$ de ingressantes do sexo feminino até o último semestre de oferta do curso. No entanto, a participação feminina é maior do que aquela observada em relação ao curso de Sistemas de Informação.

Conforme pode-se observar no gráfico, a última entrada de alunos no curso técnico em Informática oferecido na modalidade concomitante no campus Nova Friburgo aconteceu no primeiro semestre de 2014. Atualmente, o curso ainda conta com alguns alunos ativos, todos terminando o Estágio Supervisionado obrigatório para a conclusão. Os 
percentuais relativos à formatura e à evasão (calculados da mesma forma que os apresentados para o curso de graduação) mostraram que o percentual de formandos do sexo feminino em todo o curso até o primeiro semestre de 2018 corresponde à metade do percentual de formandos do sexo masculino. Já o percentual de cancelamento voluntário de matrículas pelo público feminino foi o dobro do efetuado pelo público masculino. Em relação aos percentuais de abandono e jubilamento, não houve grandes variações entre os dois públicos.

A Tabela 1 apresenta um comparativo entre o número de matrículas nos cursos técnicos ofertados no eixo tecnológico em análise na modalidade integrado a nível nacional, institucional e apenas no campus Nova Friburgo.

Tabela 1. Número de matrículas nos cursos técnicos integrados no ano de 2017

\begin{tabular}{l|c|c|c|c|c}
\hline \multirow{2}{*}{ Abrangência } & \multicolumn{3}{|c|}{ Freq. Absoluta } & \multicolumn{2}{c}{ Freq. Relativa } \\
\cline { 2 - 6 } & F & M & Total & F & M \\
\hline Nacional & 17598 & 29841 & 47439 & $37 \%$ & $63 \%$ \\
Cefet/RJ & 157 & 383 & 540 & $29 \%$ & $71 \%$ \\
$\begin{array}{l}\text { Cefet/RJ campus } \\
\text { Nova Friburgo }\end{array}$ & 41 & 77 & 118 & $35 \%$ & $65 \%$ \\
\hline
\end{tabular}

Observa-se que, embora o percentual de estudantes do sexo feminino no campus Nova Friburgo esteja abaixo do percentual nacional, o mesmo está acima do percentual institucional. Comparando os dados da tabela com aqueles apresentados na Figura 1, observa-se um percentual maior de mulheres nos cursos técnicos do que aqueles observados nos cursos de graduação do eixo tecnológico analisado.

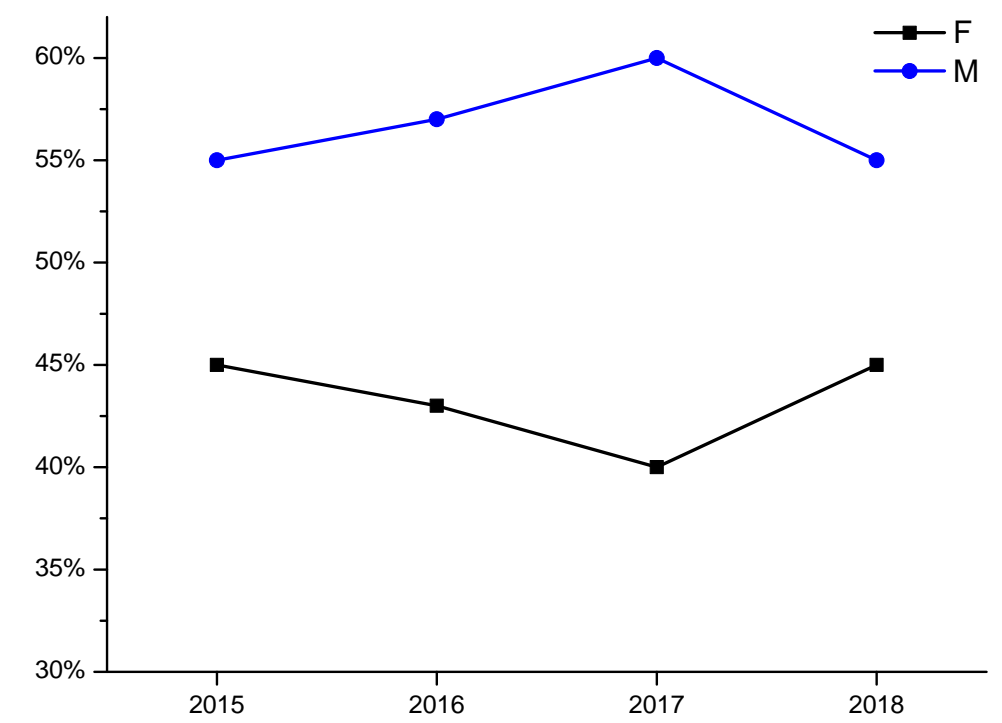

Figura 5. Percentual de ingressantes por sexo no curso Técnico de Informática integrado do "omitido para revisão"

O gráfico da Figura 5 apresenta o perfil dos ingressantes no curso técnico de Informática integrado ao Ensino Médio do campus Nova Friburgo. Neste caso, observa-se 
um percentual de ingresso de mulheres bem maior do que os apresentados anteriormente: o percentual médio nos quatro anos de curso foi de $43 \%$. Um dos possíveis fatores para esse aumento considerável é a modalidade de oferta do curso. Muitos desses alunos procuram uma oportunidade de cursar o Ensino Médio em uma escola federal devido à reconhecida qualidade do ensino oferecida pela rede, ainda que, de início, o curso técnico oferecido não desperte seu interesse.

Apresenta-se agora, Figura 6, uma análise da situação da matrícula dos alunos do curso no ano 2018 através dos dados coletados no SIE.

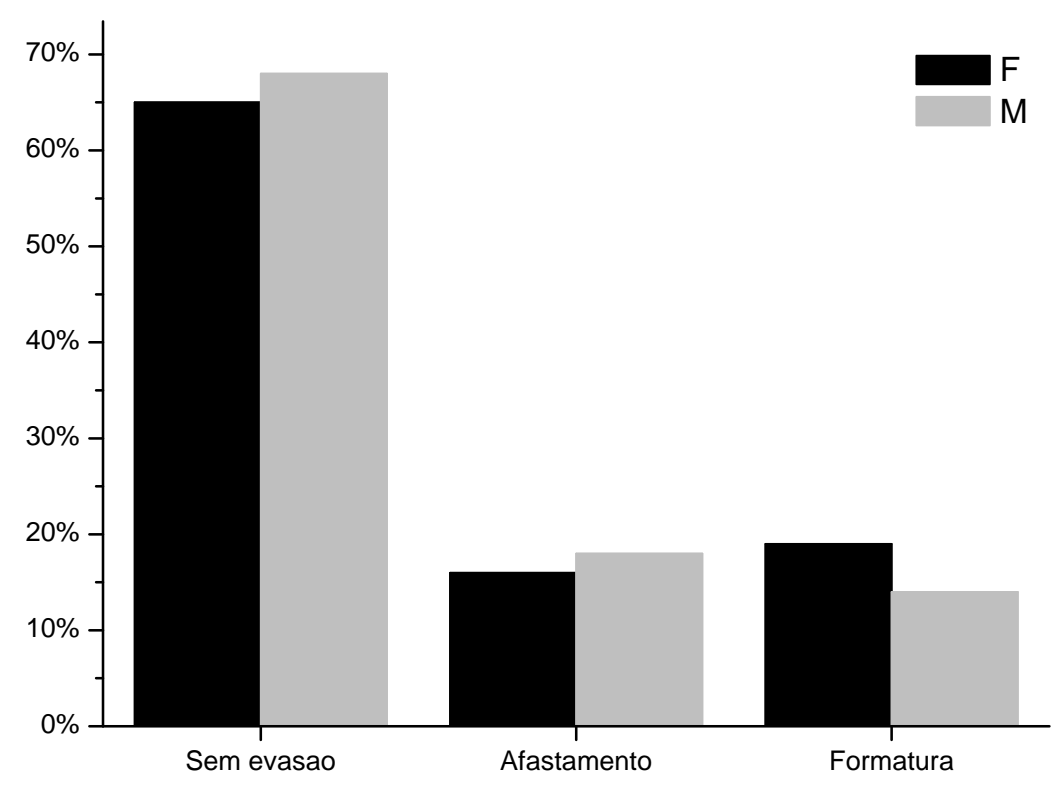

Figura 6. Percentual relativo de alunos por situação de matrícula no ano de 2018

Os percentuais apresentados no gráfico da Figura 6 foram calculados da mesma forma que aqueles apresentados na Figura 3, ou seja, os percentuais de situação de matrícula foram calculados relativamente ao número total de alunos de cada sexo. No indicador afastamento incluem-se os alunos que solicitaram transferência para outra escola ou que foram jubilados do curso. É interessante observar que, nesse caso, o percentual de formandos do sexo feminino é superior ao do sexo masculino. De fato, o curso teve apenas uma única turma formada, com o ingresso de 23 homens e de 19 mulheres no ano de 2015. No final do curso, no ano de 2017, o número de formandos dos dois sexos foi o mesmo (14 estudantes de cada sexo).

\section{Conclusões}

A diversidade social é um ponto importante a se considerar na montagem de equipes inovadoras. Segundo [Medin et al. 2014], um grupo diverso em gênero, etnia e sexo tende a ser mais inovador do que um homogêneo. Além disso, tem melhor desempenho na solução de problemas complexos por trazerem novas informações e diferentes perspectivas, mas também pela necessidade, em face de um grupo diverso, de se prepararem melhor, analisando diferentes pontos de vista e antecipando a dificuldade em se chegar a um consenso. 
A regra em Tecnologia da Informação é o trabalho em equipe, logo a importância da diversidade também se aplica. Porém, percebe-se que nessa área, a participação feminina ainda é tímida. Os dados apresentados neste artigo mostram número muito baixo de alunas matriculadas nos cursos superiores de Bacharelado e de Tecnologia e também nos cursos técnicos do eixo tecnológico de Informação e Comunicação/Informática na Rede Federal de Educação Profissional, Científica e Tecnológica e em especial nos cursos do campus Nova Friburgo.

No entanto, é interessante observar uma maior participação desse público nos cursos técnicos, principalmente na modalidade integrada ao Ensino Médio. No caso do campus Nova Friburgo, o número de formandas no curso técnico enfatiza o exposto por [Frieze et al. 2006], que afirma que a participação das mulheres em um determinado campo de estudo não pode ser atribuída ao gênero, mas sim aos aspectos culturais.

A pesquisa realizada neste trabalho, contribui como análise estratégica para o Cefet/RJ a fim de promover práticas inclusivas no cotidiano intitucional como as apresentadas por [Maciel and Bim 2016] e por [Heinzmann et al. 2016].

Cabe ressaltar que a Plataforma Nilo Peçanha ainda disponibiliza poucos dados por sexo e faixa etária dificultando uma análise mais profunda sobre a participação feminina nos cursos de nível técnico à nível regional e/ou nacional.

Como proposta de extensão deste tabalho sugere-se a realização de uma pesquisa qualitativa com as alunas de todo o Cefet/RJ, incluindo as dos cursos que não se enquadram no eixo tecnológico aqui analisado a fim de identificar os possíveis fatores sociais e culturais que levam à escolha por outras áreas, bem como com as alunas egressas do Ensino Técnico sobre o porquê das mesmas continuarem ou não na área.

\section{Referências}

Alves, H. V. S. (2016). Educação profissional e percepção de gênero: uma investigação entre alunas e alunos do Serviço Nacional de Aprendizagem Comercial SENAC de Porto Velho - RO. Revista Formação (Online), 4(23):31-56.

Brasil (2018). Plataforma Nilo Peçanha.
$<$ https://www.plataformanilopecanha.org $>$. Acesso em: 10-outubro-2018.

de Sousa, J. S., de Souza, M. C., Lopes, M. D., and da Rocha Fernandes, A. M. (2016). Mulheres Digitais: Uma análise da participação das mulheres nos cursos de Ciência da Computação e Engenharia de Computação no Brasil e na Universidade Univali. In Anais do Computer on the Beach, pages 404-413, Florianópolis, SC, Brasil.

Frieze, C., Hazzan, O., Blum, L., and Dias, M. B. (2006). Culture and environment as determinants of women's participation in computing: Revealing the "women-cs fit". SIGCSE Bull., 38(1):22-26.

Heinzmann, L., Romagna, M. E., Gosteinski, M. A. C., and da Silva, J. M. C. (2016). Meninas e jovens na computação. In Anais do Computer on the Beach, pages 554558, Florianópolis, SC, Brasil.

Hill, C., Corbett, C., and Rose, A. S. (2010). Why So Few? Women in Science, Technology, Engineering, and Mathematics. 
Maciel, C. and Bim, S. A. (2016). Programa Meninas Digitais - ações para divulgar a Computação para meninas do ensino médio. In Anais do Computer on the Beach, pages 327-336, Florianópolis, SC, Brasil.

Medin, D., Megan, C. D. L., and Bang (2014). Particular points of view. Scientific American, 311(4):44-45.

Mendes, L. B. and da Silva Figueiredo, K. (2015). Uma análise da participação feminina no curso de Sistemas de Informação da Universidade Federal de Mato Grosso. In Anais da Escola Regional de Informática da Sociedade Brasileira de Computação (SBC) - Regional de Mato Grosso, pages 62-68, Cuiabá, MT, Brasil.

Monard, M. C. and Fortes, R. P. M. (2013). Uma visão da participação feminina nos cursos de Ciência de Computação no Brasil. In Memórias del V Congreso de la Mujer Latinoamericana en la Computacion, Caracas.

Moreira, J., Mattos, G., Barreto, L., Cavaco, I., and Moreira, R. (2016). Atraindo meninas para a Ciência da Computação: Métodos e Ferramentas. In Anais dos Workshops do Congresso Brasileiro de Informatica na Educação, pages 1255-1264, Uberlândia, MG, Brasil.

NCWIT (2016). Women in Tech: The Facts. Disponível em: $<$ https://www.ncwit.org/sites/default/files/resources/ncwit_women-in-it_2016-fullreport_final-web06012016.pdf > . Acesso em: 10-outubro-2018.

Santos, W. O. (2017). Mulheres na Computação: Uma análise da participação feminina nos cursos de Licenciatura em Computação. In Anais dos Workshops do CBIE 2017, pages $814-823$, Recife, PE, Brasil.

Tabak, F. (2002). O laboratório de Pandora: Estudos sobre a ciência no feminino. Garamond, Rio de Janeiro. 